\title{
Dietary fat intake and risk of disabling hearing impairment: a prospective population-based cohort study
}

\author{
Humberto Yévenes-Briones ${ }^{1}$ (1) - Francisco Félix Caballero ${ }^{1}$ - Ellen A. Struijk ${ }^{1}$. Alberto Lana ${ }^{2}$. \\ Fernando Rodríguez-Artalejo ${ }^{1,3} \cdot$ Esther Lopez-Garcia ${ }^{1,3}$
}

Received: 9 March 2021 / Accepted: 16 July 2021 / Published online: 21 July 2021

(c) The Author(s) 2021, corrected publication 2021

\begin{abstract}
Purpose To examine the associations of specific dietary fats with the risk of disabling hearing impairment in the UK Biobank study.

Methods This cohort study investigated 105,592 participants (47,308 men and 58,284 women) aged $\geq 40$ years. Participants completed a minimum of one valid 24-h recall (Oxford Web-Q). Dietary intake of total fatty acids, polyunsaturated fatty acids (PUFA), saturated fatty acids (SFA), and monounsaturated fatty acids (MUFA) was assessed at baseline. Functional auditory capacity was measured with a digit triplet test (DTT), and disabling hearing impairment was defined as a speech reception threshold in noise $>-3.5 \mathrm{~dB}$ in any physical exam performed during the follow-up.

Results Over a median follow-up of 3.2 (SD: 2.1) years, 832 men and 872 women developed disabling hearing impairment. After adjustment for potential confounders, including lifestyles, exposure to high-intensity sounds, ototoxic medication and comorbidity, the hazard ratios (HRs), and 95\% confidence interval (CI) of disabling hearing function, comparing extreme quintiles of intakes were $0.91(0.71-1.17)$ for total fat, $1.09(0.83-1.44)$ for PUFA, $0.85(0.64-1.13)$ for SFA and 1.01 (0.74-1.36) for MUFA among men. Among women, HRs comparing extreme intakes were $0.98(0.78-1.24)$ for total fat, 0.69 (0.53-0.91) for PUFA, $1.26(0.96-1.65)$ for SFA, and $0.91(0.68-1.23)$ for MUFA. Replacing 5\% of energy intake from SFA with an equivalent energy from PUFA was associated with 25\% risk reduction (HR: 0.75; 95\% CI: 0.74-0.77) among women. Conclusions PUFA intake was associated with decreased risk of disabling hearing function in women, but not in men.
\end{abstract}

Keywords Dietary fat intake $\cdot$ Hearing function $\cdot$ UK Biobank $\cdot$ Cohort study

\section{Introduction}

Hearing loss is one of the main leading causes of years lived with disability; moreover, it has been considered an "invisible disability", since it is usually underestimated in comparison with other health problems [1]. In older people,

Humberto Yévenes-Briones

humbertoyevenesbriones@gmail.com

Esther Lopez-Garcia

esther.lopez@uam.es

1 Department of Preventive Medicine and Public Health, School of Medicine, Universidad Autónoma de MadridIdiPaz and CIBERESP (CIBER of Epidemiology and Public Health), C/ Arzobispo Morcillo, s/n, 28029 Madrid, Spain

2 Department of Medicine, School of Medicine and Health Sciences, Universidad de Oviedo /ISPA, Oviedo, Spain

3 IMDEA-Food Institute, CEI UAM+CSIC, Madrid, Spain hearing loss has been associated with higher risk of social isolation [2], depression [3], cognitive impairment [4], poor quality of life [5], and also with higher risk of cardiovascular disease and all-cause mortality [6-8]. In addition, a large body of evidence suggests that sex modulates susceptibility to age-related hearing loss, because of the protective effect of estrogens on hearing function and differences in the processing of stimuli at the cortical level [9].

Besides age-associated biological degeneration and noise exposure, one mechanism of hearing loss is impaired vascular function. The cochlea of the inner ear is highly vascularized and supported by a single artery; thus, dietary exposures that are able to improve vascular function may have a protective role on hearing capacity. However, the evidence on the effect of habitual diet on hearing loss is limited $[10,11]$. High intake of some nutrients, such as $\beta$-carotene, $\beta$-cryptoxanthin, vitamin $B 12$, folic acid, vitamin $D$, and magnesium, have been associated with lower risk of hearing 
loss [12-15]. As regards macronutrient intake, three studies have focused on the effect of polyunsaturated fatty acids (PUFA). They found that a higher intake of n-3 PUFA was associated with decreased hearing loss risk [16-18]. In addition, a cross-sectional study found that low fat and protein intakes were associated with hearing impairment [19].

There is compelling evidence that different types of dietary fats have opposed effects on cardiovascular disease by modifying serum lipid profiles, endothelial function, chronic inflammation, and blood clotting, and that the type of fat is more important than the total amount ingested [20]. Similarly, different associations have been found between distinct types of dietary fats and risk of frailty [21] or physical function impairment [22]. Understanding the effect of habitual intake of specific fatty acids on hearing function could help to develop dietary recommendations for healthy aging, including optimal hearing. Therefore, the objective of this study was to examine the prospective association of fatty acids intake with disabling hearing impairment, assessed through the functional auditory capacity, in a large population-based study of middle and older-age men and women of the UK.

\section{Methods}

\section{Study design and participants}

The UK Biobank study is a large population-based cohort study established in 2006-2010 throughout the United Kingdom [23]. The study recruited more than 500,000 participants aged between 40 and 70 years, who gave information on health status, demographics, and lifestyle. In addition, they provided several types of biological samples and underwent a physical examination. Participants were followed to update information in 2012-2013 and in 2014-2016.

\section{Dietary assessment}

Food consumption was collected with five 24 -h recalls (Oxford Web-Q), a detailed computerized questionnaire on the intake of 200 commonly consumed foods and beverages in the previous $24 \mathrm{~h}[24,25]$. Unlike standard 24-h diet recalls, where the respondents are asked to remember and report the food consumed, the Web-Q presents 21 food groups and asks the participants if they consumed any of them over the previous day. Positive answers open additional questions in which participants have to select the type of food consumed and its amount, based on standard serving categories or portions. Thus, the data collection approach used in this tool could be defined as a hybrid between a 24-h dietary recall and a food frequency questionnaire [24]. The Web-Q automatically calculated nutrient intakes from food composition tables specific for the United Kingdom [26]. Since we focused on average macronutrient intakes, which are stable to day-to-day variation on food consumption [27], we included those participants who completed at least one Web-Q, and calculated the average nutrient intake from all available Web-Qs. For the calculation of monounsaturated fatty acids (MUFA), we subtracted saturated (SFA) and PUFA from the total fat intake [28]. Intake of subtypes of PUFA, trans fat, and dietary cholesterol were not available. Total and specific fatty acids intake was expressed as a percentage of ingested energy. In a recent publication, main sources of total fats have been identified as the following food groups: "desserts and cakes and pastries", "high fat cheese", "dairy fat spread", "egg and egg dishes", and "biscuits"; main sources of SFA were "high fat cheese", "desserts and cakes and pastries", "dairy fat spread", "milk-dairy desserts", and "biscuits". No information of main sources of PUFA was available [29]. Finally, overall diet quality was assessed by adherence to the alternate Mediterranean Diet score (aMED), after excluding the component score for fatty acids [30].

\section{Functional auditory capacity and hearing-related variables}

Functional auditory capacity was measured with a digit triplet test (DTT) to determine the speech reception threshold noise (SRTn). The SRTn is a measure of the ability to understand speech in noise. Before starting the test, participants were asked to remove their hearing aid if they had it. In addition, the volume of the speech was set to the individual's most comfortable level for each ear. Then, the participant listened to 15 sets of three digits presented with background noise and had to enter each triplet on a keyboard on the touch screen. If the triplet was correctly identified, the noise level was increased for the next triplet; otherwise, the noise level was decreased. Each ear was tested separately, and SRTn was defined as the signal-to-noise-ratio at which half of the presented digits could be recognized correctly. The signal-to-noise-ratio could range between -12 and $+8 \mathrm{~dB}$. In the analyses, we used the SRTn for the best ear in each participant in both measurements, at baseline and at the follow-up, and if the SRTn was only available for one ear, we assumed that it was the best one. Dawes et al. [31] have established the cut-off points to categorize the UK Biobank population as with normal (SRTn $<-5.5 \mathrm{~dB}$ ), insufficient (SRTn $\geq-5.5$ to $\leq-3.5 \mathrm{~dB}$ ) or poor hearing function (SRTn $>-3.5 \mathrm{~dB}$ ). We defined disabling hearing impairment as a SRTn $>-3.5 \mathrm{~dB}$ in any physical exam during the follow-up. The DTT has shown a good correlation with pure-tone audiometry $(r=0.77)$, which suggests that about $60 \%$ of the performance on DTT is explained by standardized audiometric data [32]. The differences in the 
psychoacoustic ability of the listeners influence the ability to recognize speech in noise, which explains the remaining variation [33].

Several hearing-related variables were also obtained. Loud music exposure, noisy workplace, and tinnitus were, respectively, assessed by asking the participants: "Have you ever listened to music for more than $3 \mathrm{~h}$ per week at a volume which you would need to shout to be heard or, if wearing headphones, someone else would need to shout for you to hear them?"; "Have you ever worked in a noisy place where you had to shout to be heard?"; and "Do you get or have you had noises (such as ringing or buzzing) in your head or in one or both ears that lasts for more than five minutes at a time?" [34].

\section{Mortality}

All-cause mortality was obtained from death certificates held by the National Health Service Information Centre (England and Wales) and the National Health Service Central Register Scotland (Scotland) [35].

\section{Other variables}

Baseline information included age, sex, ethnicity, educational level, and smoking status. Weight and height were also measured under standardized conditions, and body mass index (BMI) was calculated as weight $(\mathrm{kg})$ divided by height (m) squared. Physical activity (metabolic equivalent tasks-hours/week, METs-h/wk) was evaluated with the Short International Physical Activity Questionnaire [36]. Cognitive function was assessed through the reaction time test, by showing 12 rounds of pairs of cards to each participant, who had to press a button as quickly as possible if both cards were the same. The test allows to calculate the average reaction time (milliseconds) of each participant to identify the pairs of cards; a longer time indicated a worse cognitive status [37]. Finally, diagnoses of diabetes, vascular or heart problems, hypercholesterolemia, or cancer and use of ototoxic medication were reported by the participants.

\section{Statistical analysis}

On a sample of 211,013 participants with dietary information, we excluded 1,023 with unrealistic energy intake $(<800$ or $>5,000 \mathrm{kcal} /$ day for men, and $<500$ or $>4000 \mathrm{kcal} /$ day for women), 103,075 without a hearing test at baseline, and 1323 with disabling hearing at baseline, leaving a total of 105,592 participants for the analysis $(47,308$ men and 58,284 women).

Participants were classified into quintiles of percentage of energy from total fat, PUFA, SFA, and MUFA. We used the analysis of variance (ANOVA) or Chi-square test, depending on the type of variable (continuous or categorical ones, respectively), to assess differences in sociodemographic characteristics, lifestyle, and morbidity across the quintiles of total fatty acid intake.

Person-years of follow-up were calculated from the first interview at baseline until the occurrence of disabling hearing impairment, death, loss to follow-up, or the end of the study (December 2016), whichever came first. We used Cox proportional hazard models to calculate hazard ratios (HRs), and their 95\% confidence intervals (CI), for the association between each quintile of total fat and PUFA, SFA, and MUFA intake and disabling hearing impairment, adjusting for potential confounders. Two types of models were built. The first model was adjusted for age. A second model was additionally adjusted for ethnic background (British/other), educational level (primary education or less, secondary education, and university degree), tobacco consumption (current smoker, former smoker, never smoker), BMI $(<25.0$, $25.0-29.9, \geq 30.0 \mathrm{~kg} / \mathrm{m}^{2}$ ), physical activity (quintiles of METs hour/week), alcohol consumption (quintiles of $\mathrm{g} / \mathrm{d}$ ), loud music exposure (yes/no), noisy workplace (no, for less than one year, for around $1-5$ years, for more than 5 years), presence of tinnitus, aspirin and ibuprofen consumption (yes/no), reaction time in cognitive function test (ms), hypercholesterolemia, vascular or heart problems, cancer, diabetes, total energy (quintiles of $\mathrm{kcal} /$ day) and protein intake, and the other fatty acids intake (quintiles of \% energy), as appropriate. Thus, the coefficient for dietary fat reflects the effect of substituting an equal amount of energy from fat for carbohydrate. Tests for linear trend were conducted by assigning the median value to each quintile and treating this as a continuous variable in the regression models. To test nonlinear risk trends, we used three knot restricted cubic splines for the consumption of specific fatty acids and the risk of disabling hearing function [38].

We performed separate analyses in women and men, as we found a statistically significant interaction term for sex and intake of PUFA when predicting incident hearing impairment $(P=0.03)$. Also, and according to the previous literature, we performed analyses stratified by subgroups of age [39], presence of tinnitus [40], being overweight or obese [41], having chronic diseases [42], and diet quality [30]. Additionally, we conducted separate analyses among those with optimal hearing at the start of the study, to understand whether the effect of fatty acids depends on the baseline hearing status. Finally, we evaluated the effect of substituting one type of dietary fat for an equal amount of another type of fat on hearing impairment. To fit these isocaloric energy density models, we simultaneously included total energy intake and the percentage of energy derived from the types of fats of interest as continuous variables (per 5\% increase), along with the covariates listed above, and calculated the difference in coefficients. Additionally, 
we modeled the substitution of each type of dietary fat for carbohydrates and for total protein.

Analyses were performed with Stata (version 15.0; Stata Corp., College Station). This manuscript follows the Strengthening the Reporting of Observational Studies in Epidemiology (STROBE) recommendations.

\section{Results}

In this cohort, mean (SD) intakes of fatty acids were 14.3 (29.8) $\mathrm{g} / \mathrm{d}$ for PUFA, 29.5 (12.8) g/d for SFA, and 33.2 (13.2) $\mathrm{g} / \mathrm{d}$ for MUFA. The percentages of energy provided for each specific fat were $6.1,12.5$, and $14.1 \%$, respectively. Baseline characteristics of the population by quintiles of total fat and sex are presented in Table 1. Compared to participants in the lowest quintile of total fat intake, those in the highest quintile had lower prevalence of primary or less education, were more likely to smoke, reported lower physical activity, were more likely exposed to loud music, and had higher cognitive performance. Ibuprofen consumption was higher among those with the highest intake, but hypercholesterolemia and vascular or heart problems were less prevalent in that group. Protein and carbohydrate intakes decreased across quintiles of increased fat intake.

Over a median follow-up of 3.2 years, 1704 (1.61\%) participants developed disabling hearing impairment [832 (1.76\%) men and 872 (1.50\%) women]. Among men, in fully adjusted models, no significant associations were found between fatty acids intake and hearing impairment: HR $(95 \%$ CI) for quintile 5 vs. 1 were $0.91(0.71-1.17)$ for total fat; $1.09(0.83-1.44)$ for PUFA; $0.85(0.64-1.13)$ for SFA; and 1.01 (0.74-1.36) for MUFA (Table 2). Among women, although no association was observed between total fat intake and disabling hearing impairment, an inverse association was found for PUFA, (HR quintile 5 vs. 1: 0.69; $95 \%$ CI: 0.53-0.91). On the contrary, SFA and MUFA intakes were not associated with the outcome (Table 2).

The dose-response association between the fatty acids and disabling hearing impairment was assessed in Fig. 1. For PUFA, an inverse association was found in women, becoming statistically significant with intakes $\geq 15 \%$ of energy. We estimated that replacing 5\% of energy intake from SFA with an equivalent energy from PUFA was associated with $25 \%$ risk reduction (HR: 0.75; 95\% CI: 0.74-0.77). Replacing 5\% of energy intake from MUFA for PUFA marginally reduced the risk (HR: 0.97; 95\% CI: 0.94-1.00), while substitution of carbohydrates and total protein for PUFAs also showed a risk reduction (Supplemental Table 1).

The results were similar when they were restricted to participants with optimal hearing at baseline (Supplemental Table 2). In stratified analyses, women with the highest PUFA intake and $<60 \mathrm{y}$, without tinnitus, with BMI $\geq 25 \mathrm{~kg} /$ $\mathrm{m}^{2}$, with chronic diseases, or with low adherence to a Mediterranean diet pattern had lower risk of disabling hearing impairment ( $p$ for interaction non-significant in any case), compared with those with the lowest intake and none of these characteristics. For SFA, we observed a higher risk for those women with the highest intake and $\geq 60 \mathrm{y}$, with tinnitus, $\mathrm{BMI}<25 \mathrm{~kg} / \mathrm{m}^{2}$ or chronic diseases ( $\mathrm{p}$ for interaction non-significant). For MUFA, no association was found in these analyses (Fig. 2).

\section{Discussion}

In this large population-based cohort study, we found that energy intake $>15 \%$ from PUFA was associated with lower risk of disabling hearing impairment among women. Replacing 5\% of energy intake from SFA for PUFA was associated with a significant reduced risk. By contrast, no association was observed among men.

Gopinath et al. [17], using data of 2000 participants in the Blue Mountains Hearing Study, found that higher increase in n-3 PUFA was associated with reduced incident hearing loss. Hearing loss was defined as hearing impairment $>25 \mathrm{~dB}$ in the pure-tone average of audiometric hearing thresholds at $500,1000,2000$, and $4000 \mathrm{~Hz}$, at the 5-year follow-up examination. In addition, Curhan et al. [16] found that, among 60,000 women from the Nurses' Health Study, those with an n-3 PUFA intake on the highest vs. lowest quintile of the distribution had a lower risk of self-reported hearing loss (RR: 0.85; 95\% CI: 0.80-0.91). Both studies suggested that the plausible beneficial effect of these fats could be due to the hypolipidemic, triglyceride lowering, and anti-inflammatory and antiatherothrombotic properties of some PUFA, which help to maintain adequate vascular supply to the cochlea [43]. However, in a recent cross-sectional study, with plasma concentrations of $n-3$ and $n-6$ PUFAs measured in 534 participants, no clear link was found between plasma levels of PUFAs and hearing function, assessed using audiometric measures [44].

The causes of hearing function impairment are multiple. The main ones include the exposure to environmental risk factors, age-associated degenerative processes, and chronic diseases that alter blood supply [45]. If there is inadequate irrigation of the auditory system, cochlear function could be affected. Alterations in the vascularization of the auditory system could hinder the evacuation of waste from cellular metabolism, that leads to the development of microvascular diseases [46]. It has been observed that an increase in PUFA intake through diet could reduce pathologies related to microvascularization [42], including impaired renal function [47].

We found a statistically significant interaction between the PUFA intake and sex on the risk of disabling hearing 


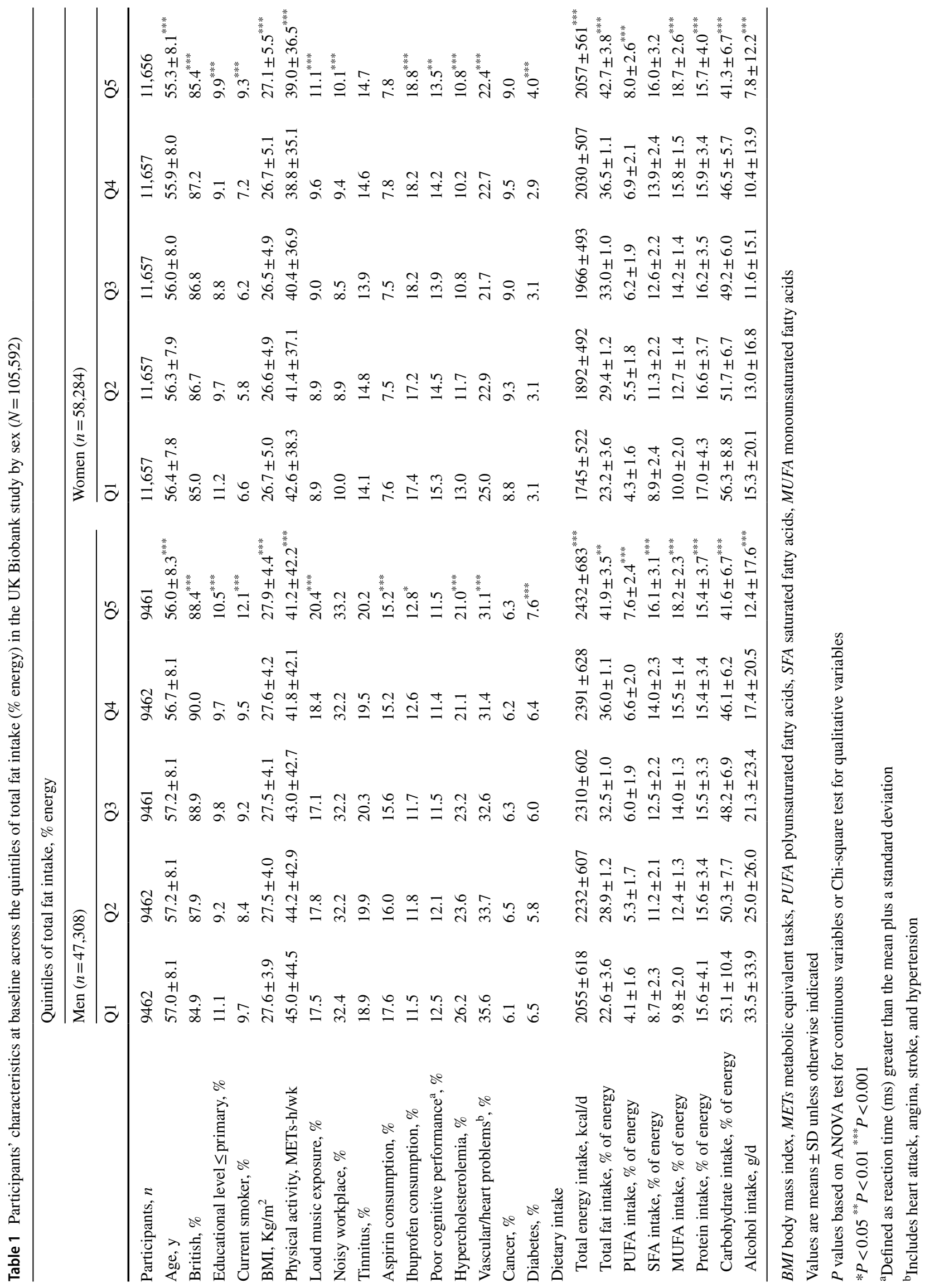


Table 2 Association between dietary fat intake (\% energy) and risk of disabling hearing function in the UK Biobank study by sex $(N=105,592)$

\begin{tabular}{|c|c|c|c|c|c|c|}
\hline & Quintile 1 & Quintile 2 & Quintile 3 & Quintile 4 & Quintile 5 & $P$ trend \\
\hline \multicolumn{7}{|l|}{ Men } \\
\hline \multicolumn{7}{|l|}{ Total fat intake } \\
\hline Participants, $n$ & 9462 & 9462 & 9461 & 9462 & 9461 & \\
\hline Range, \% energy & $1.6-26.7$ & $26.8-30.7$ & $30.8-34.1$ & $34.2-37.9$ & $38.0-68.6$ & \\
\hline Person-yr & 36,053 & 37,404 & 37,557 & 37,400 & 36,815 & \\
\hline Cases, $n$ & 151 & 184 & 196 & 159 & 142 & \\
\hline Age-adjusted model & 1.00 & $1.01(0.82-1.26)$ & $1.07(0.87-1.33)$ & $0.90(0.72 .1 .12)$ & $0.89(0.70-1.11)$ & 0.17 \\
\hline MV-adjusted model & 1.00 & $1.04(0.83-1.29)$ & $1.11(0.89-1.39)$ & $0.94(0.74-1.19)$ & $0.91(0.71-1.17)$ & 0.31 \\
\hline \multicolumn{7}{|l|}{ PUFA intake } \\
\hline Participants, $n$ & 9462 & 9462 & 9461 & 9462 & 9461 & \\
\hline Range, \% energy & $0.8-3.9$ & $4.0-5.1$ & $5.2-6.2$ & $6.3-7.6$ & $7.7-18.7$ & \\
\hline Person-yr & 35,766 & 37,610 & 37,642 & 37,612 & 36,599 & \\
\hline Cases, $n$ & 130 & 179 & 180 & 198 & 145 & \\
\hline Age-adjusted model & 1.00 & $1.09(0.87-1.37)$ & $1.12(0.89-1.40)$ & $1.22(0.98-1.52)$ & $1.07(0.84-1.35)$ & 0.45 \\
\hline MV-adjusted model & 1.00 & $1.11(0.88-1.40)$ & $1.15(0.90-1.46)$ & $1.24(0.97-1.59)$ & $1.09(0.83-1.44)$ & 0.48 \\
\hline \multicolumn{7}{|l|}{ SFA intake } \\
\hline Participants, $n$ & 9462 & 9462 & 9461 & 9462 & 9461 & \\
\hline Range, $\%$ energy & $0.3-9.5$ & $9.6-11.4$ & $11.5-13.1$ & $13.2-15.2$ & $15.3-30.5$ & \\
\hline Person-yr & 36,419 & 37,134 & 37,558 & 37,432 & 36,686 & \\
\hline Cases, $n$ & 166 & 185 & 171 & 173 & 137 & \\
\hline Age-adjusted model & 1.00 & $1.01(0.82-1.25)$ & $0.89(0.72-1.10)$ & $0.93(0.75-1.15)$ & $0.81(0.65-1.02)$ & 0.05 \\
\hline MV-adjusted model & 1.00 & $1.04(0.83-1.29)$ & $0.91(0.72-1.15)$ & $0.97(0.76-1.24)$ & $0.85(0.64-1.13)$ & 0.23 \\
\hline \multicolumn{7}{|l|}{ MUFA intake } \\
\hline Participants, $n$ & 9462 & 9462 & 9461 & 9462 & 9461 & \\
\hline Range, \% energy & $0.6-11.3$ & $11.4-13.1$ & $13.2-14.7$ & $14.8-16.5$ & $16.6-37.0$ & \\
\hline Person-yr & 35,927 & 37,412 & 37,481 & 37,414 & 36,995 & \\
\hline Cases, $n$ & 145 & 187 & 180 & 161 & 159 & \\
\hline Age-adjusted model & 1.00 & $1.05(0.84-1.30)$ & $1.00(0.80-1.25)$ & $0.93(0.74-1.16)$ & $0.97(0.77-1.21)$ & 0.48 \\
\hline MV-adjusted model & 1.00 & $1.03(0.81-1.30)$ & $1.02(0.79-1.31)$ & $0.95(0.72-1.26)$ & $1.01(0.74-1.36)$ & 0.87 \\
\hline \multicolumn{7}{|l|}{ Women } \\
\hline \multicolumn{7}{|l|}{ Total fat intake } \\
\hline Participants, $n$ & 11,657 & 11,657 & 11,657 & 11,657 & 11,656 & \\
\hline Range, $\%$ energy & $1.6-27.2$ & $27.3-31.2$ & $31.3-34.6$ & $34.7-38.5$ & $38.6-70.9$ & \\
\hline Person-yr & 43,174 & 44,272 & 44,430 & 44,633 & 43,703 & \\
\hline Cases, $n$ & 152 & 185 & 186 & 187 & 162 & \\
\hline Age-adjusted model & 1.00 & $1.05(0.85-1.30)$ & $1.04(0.84-1.29)$ & $1.04(0.84-1.29)$ & $1.02(0.82-1.27)$ & 0.90 \\
\hline MV-adjusted model & 1.00 & $1.04(0.84-1.30)$ & $1.02(0.82-1.27)$ & $1.01(0.81-1.26)$ & $0.98(0.78-1.24)$ & 0.79 \\
\hline \multicolumn{7}{|l|}{ PUFA intake } \\
\hline Participants, $n$ & 11,657 & 11,657 & 11,657 & 11,657 & 11,656 & \\
\hline Range, \% energy & $0.03-4.0$ & $4.1-5.2$ & $5.3-6.4$ & $6.5-7.9$ & $8.0-25.3$ & \\
\hline Person-yr & 42,908 & 44,465 & 44,530 & 44,370 & 43,939 & \\
\hline Cases, $n$ & 168 & 184 & 183 & 204 & 133 & \\
\hline Age-adjusted model & 1.00 & $0.89(0.72-1.09)$ & $0.88(0.71-1.08)$ & $1.01(0.82-1.24)$ & $0.69(0.55-0.87)$ & 0.01 \\
\hline MV-adjusted model & 1.00 & $0.86(0.69-1.07)$ & $0.84(0.67-1.06)$ & $0.98(0.77-1.23)$ & $0.69(0.53-0.91)$ & 0.04 \\
\hline \multicolumn{7}{|l|}{ SFA intake } \\
\hline Participants, $n$ & 11,657 & 11,657 & 11,657 & 11,657 & 11,656 & \\
\hline Range, \% energy & $0.1-9.6$ & $9.7-11.5$ & $11.6-13.2$ & $13.3-15.2$ & $15.3-33.8$ & \\
\hline Person-yr & 42,963 & 44,328 & 44,530 & 44,532 & 43,859 & \\
\hline Cases, $n$ & 127 & 201 & 187 & 182 & 175 & \\
\hline Age-adjusted model & 1.00 & $1.34(1.07-1.67)$ & $1.23(0.99-1.55)$ & $1.22(0.97-1.53)$ & $1.27(1.01-1.59)$ & 0.17 \\
\hline
\end{tabular}


Table 2 (continued)

\begin{tabular}{|c|c|c|c|c|c|c|}
\hline & Quintile 1 & Quintile 2 & Quintile 3 & Quintile 4 & Quintile 5 & $P$ trend \\
\hline MV-adjusted model & 1.00 & $1.36(1.08-1.71)$ & $1.24(0.97-1.58)$ & $1.19(0.93-1.53)$ & $1.26(0.96-1.65)$ & 0.36 \\
\hline \multicolumn{7}{|l|}{ MUFA intake } \\
\hline Participants, $n$ & 11,657 & 11,657 & 11,657 & 11,657 & 11,656 & \\
\hline Range, $\%$ energy & $0.8-11.5$ & $11.6-13.3$ & $13.4-14.9$ & $15.0-16.8$ & $16.9-38.7$ & \\
\hline Person-yr & 43,137 & 44,325 & 44,656 & 44,551 & 43,543 & \\
\hline Cases, $n$ & 157 & 182 & 202 & 190 & 144 & \\
\hline Age-adjusted model & 1.00 & $1.03(0.83-1.27)$ & $1.09(0.88-1.34)$ & $1.04(0.84-1.29)$ & $0.90(0.72-1.13)$ & 0.42 \\
\hline MV-adjusted model & 1.00 & $1.04(0.82-1.31)$ & $1.09(0.85-1.38)$ & $1.03(0.79-1.33)$ & $0.91(0.68-1.23)$ & 0.51 \\
\hline
\end{tabular}

PUFA polyunsaturated fatty acids, SFA saturated fatty acids, MUFA monounsaturated fatty acids

Values are hazard ratios ( $95 \%$ confidence interval)

Multivariable (MV) adjusted model: Cox regression model adjusted for age, ethnic background, educational level ( $\leq$ primary, secondary, university), tobacco (current smoker, former smoker, never smoker), BMI ( $\left.<25.0,25.0-29.9, \geq 30.0 \mathrm{~kg} / \mathrm{m}^{2}\right)$, physical activity (quintiles of METs-h/ wk), alcohol consumption (quintiles of $\mathrm{g} / \mathrm{d}$ ), loud music exposure (yes/no), noisy workplace (no, for less than a year, for around 1-5 years, for more than 5 years), tinnitus, aspirin, ibuprofen consumption, reaction time (ms), hypercholesterolemia, vascular/heart problems, cancer, diabetes, total energy (quintiles of $\mathrm{kcal} / \mathrm{day}$ ), and protein intake (quintiles of \% energy). Models for PUFA were adjusted for SFA and MUFA and vice versa (quintiles of $\%$ energy)

impairment. Some studies suggest that estrogens could protect hearing function $[9,48]$. There is also evidence of sex differences in the reception of complex stimuli such as speech, possibly due to differences in the activation of language processing at the cortical level, because of the distinct length of the cochlea in men and women [49]. Understanding the biological pathways that explain sex differences associated with nutritional exposures on health outcomes seems necessary to develop targeted strategies for the population [50].

Stratified analyses were robust showing a reduced risk associated with higher intake of PUFA in women. We observed stronger associations in women younger than 60 years; this is biologically plausible, since at increasing age, the ability to identify speech stimuli decreases greatly and beneficial dietary effects may be insufficient to reverse this situation [51]. We also observed a stronger inverse association among women without tinnitus. Since tinnitus and hearing loss are strongly related, and PUFA intake has also been related to tinnitus [19], it is unclear if tinnitus can partly mediate in this association. Furthermore, the very strong associations found in those with excess weight or having chronic diseases may indicate that common biological mechanisms underlie hearing impairment, obesity, and chronic diseases [39, 52].

Regarding our findings on the intake of SFA, we observed a higher risk of hearing impairment associated to consumptions above $10 \%$ of energy intake. Although we could not find a dose-response association, substitution analyses showed that replacing PUFA for SFA decreased the risk of disabling hearing loss; more research is needed to understand the role of SFA in hearing function. In addition, replacing PUFA for MUFA was also associated with a slight reduction of risk. A recent paper has suggested that MUFA from sources other than olive oil (e.g., red meats and high-fat dairy products) may have a detrimental effect on health [53].

The strengths of our study lie in its prospective design and large sample size. Besides, the hearing measurement test includes not only pure tones but also speech in noise that simultaneously scans many frequencies of human speech, especially those close to $1000 \mathrm{~Hz}$. Furthermore, our analyses were adjusted for many potential confounders, such as lifestyle, exposure to high-intensity sounds, ototoxic medication, and morbidity. The study also has several limitations, including the use of 24-h-recall questionnaires to estimate habitual diet. We could not explore the separate association of different PUFA, including $n-3$ and $n-6$, or MUFA from plant vs. animal sources with hearing function due to the lack of this information in the database analyzed. Specifically, since n-6 PUFA are the main source of PUFA in most populations, including the cohort analyzed here, we cannot discard that most of the effect observed is due to this fatty acid instead of $n-3$ PUFA, in contrast to the previous studies. Although the role of n-6 PUFA in inflammation has been controversial, our previous findings based on the effect of these fatty acids on plasma concentration of inflammatory biomarkers [54] and others on circulating arachidonic acid levels [55] suggest that dietary n-6 PUFA do not increase inflammatory processes and may help improving endothelial function and chronic inflammation.

In conclusion, higher consumption of PUFA was associated with decreased risk of disabling hearing function in women. Replacing 5\% of total energy intake from SFA by the same energy from dietary PUFA may contribute to delay hearing loss. Further research may help understanding the differences found in men and women and which types 

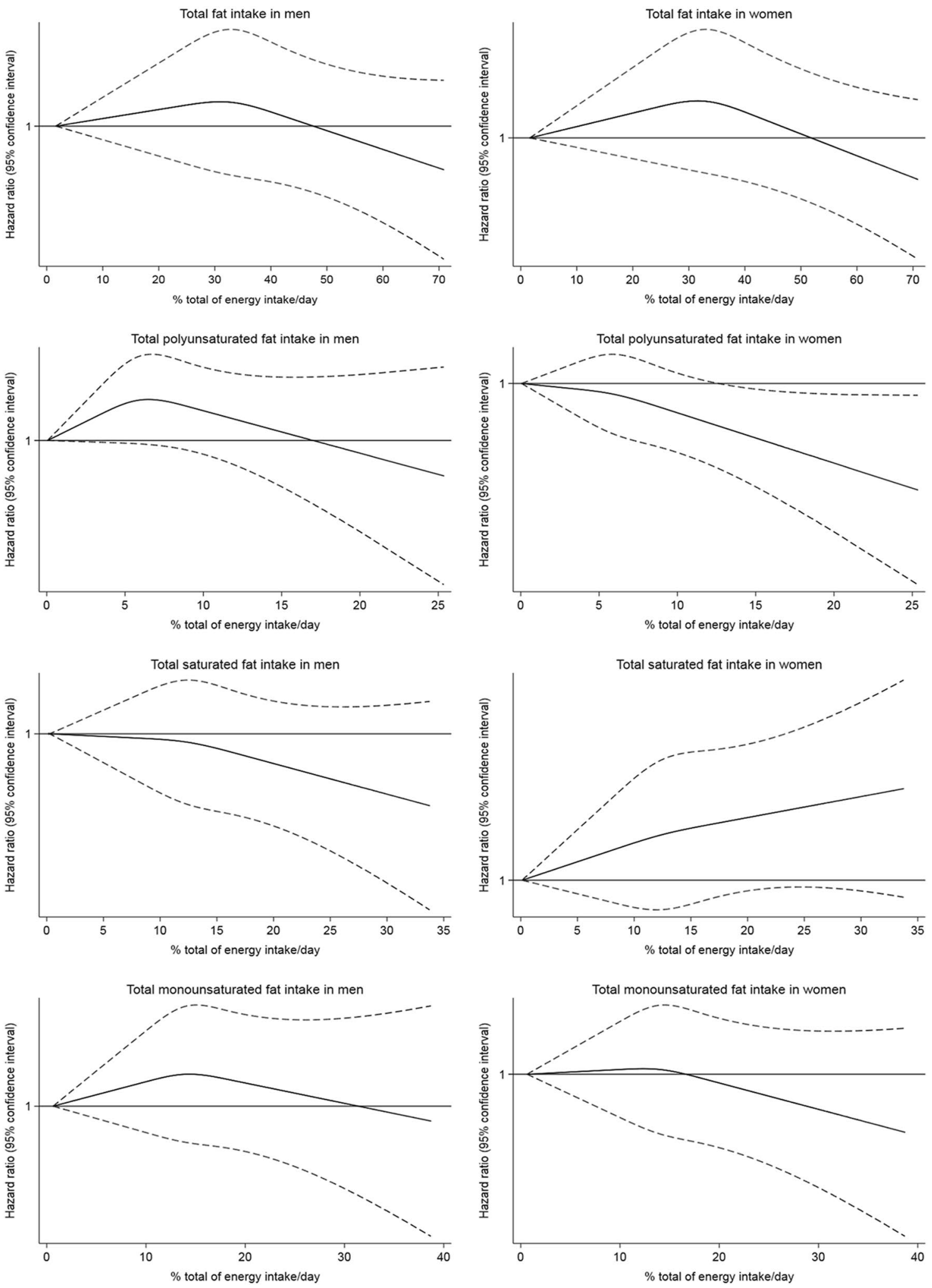

Fig. 1 Multivariable adjusted spline curves for the relation between dietary fat intake and the risk of disabling hearing function in men and women. Covariates were age, ethnic background, educational level ( $\leq$ primary, secondary, university), tobacco (current smoker, former smoker, never smoker), BMI $(<25.0,25.0-29.9, \geq 30.0 \mathrm{~kg} /$ $\mathrm{m}^{2}$ ), physical activity (quintiles of METs-h/wk), alcohol consumption

(quintiles g/d), loud music exposure (yes/no), noisy workplace (no, for less than a year, for around 1-5 years, for more than 5 years), tinnitus, aspirin, ibuprofen consumption, reaction time (ms), hypercholesterolemia, vascular/heart problems, cancer, diabetes, total energy (quintiles of $\mathrm{kcal} / \mathrm{day}$ ), and protein intake (quintiles of $\%$ energy), and for PUFA, SFA, and MUFA (in quintiles of \% energy), as appropriate 
Polyunsaturated fat intake in men

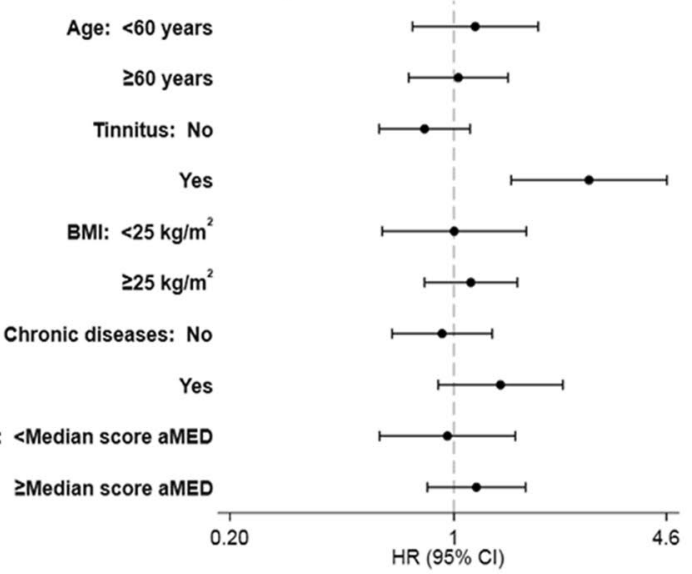

Saturated fat intake in men

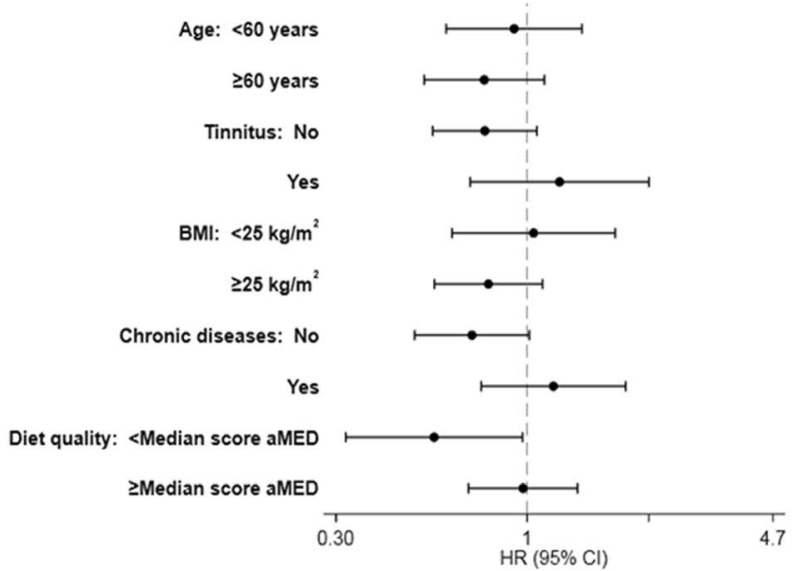

Monounsaturated fat intake in men

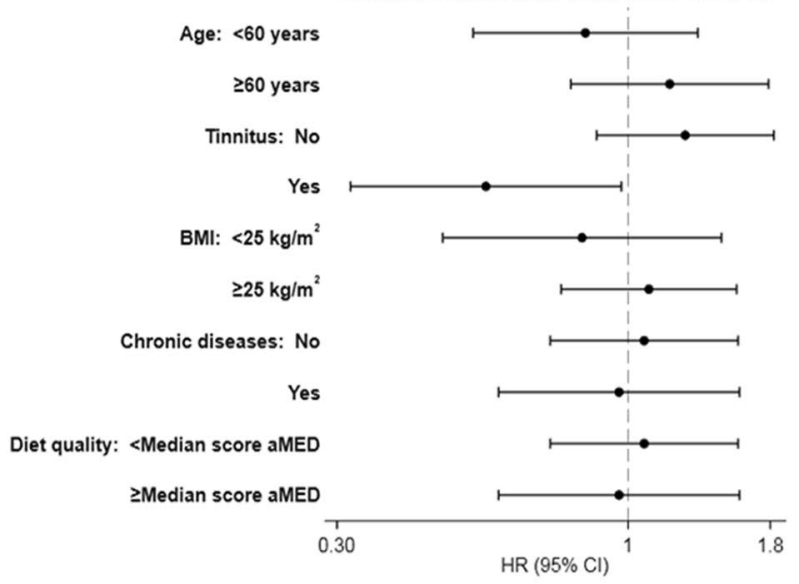

Fig. 2 Hazard ratios (95\% confidence interval) for the association between dietary fat intake and risk of disabling hearing function in the UK Biobank study stratified by sex in subgroups of participants (quintile of highest intake compared to the quintile of lowest intake). Cox regression model adjusted for age, ethnic background, educational level ( $\leq$ primary, secondary, university), tobacco (current smoker, former smoker, never smoker), BMI $(<25.0,25.0$ $29.9, \geq 30.0 \mathrm{~kg} / \mathrm{m}^{2}$ ), physical activity (quintiles of METs-h/wk), alco-
Polyunsaturated fat intake in women

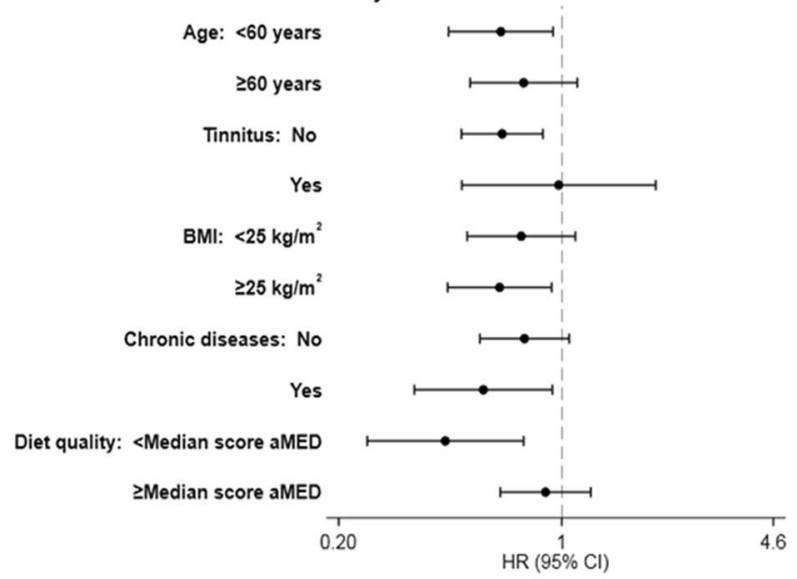

Saturated fat intake in women
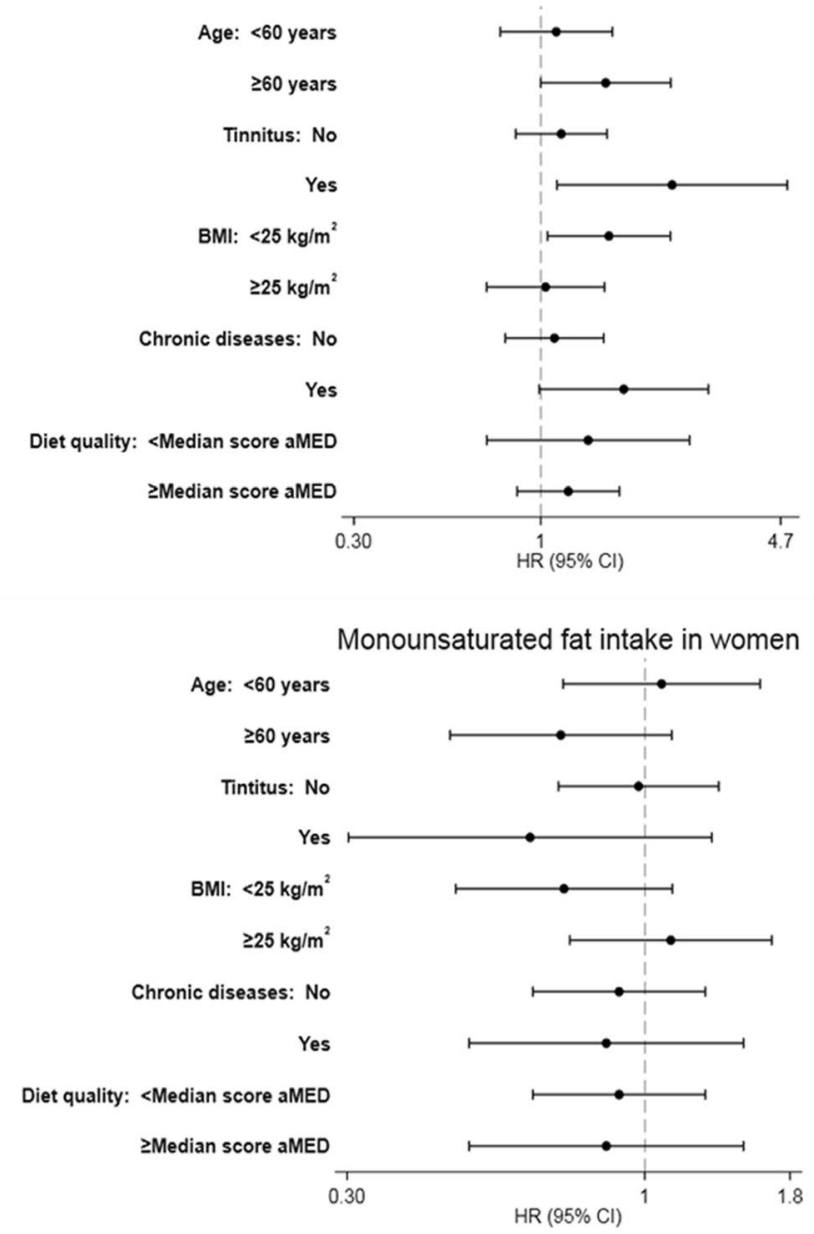

hol consumption (quintiles g/d), loud music exposure (yes/no), noisy workplace (no, for less than a year, for around 1-5 years, for more than 5 years), tinnitus, aspirin, ibuprofen consumption, reaction time (ms), hypercholesterolemia, vascular/heart problems, cancer, diabetes, total energy (quintiles of $\mathrm{kcal} / \mathrm{day}$ ), protein intake (quintiles of $\%$ energy), and for PUFA, SFA, and MUFA (in quintiles of $\%$ energy), as appropriate 
of PUFAs are contributing to this association; also, other studies should elucidate if SFA intake increases the risk of hearing loss.

Supplementary Information The online version contains supplementary material available at https://doi.org/10.1007/s00394-021-02644-7.

Acknowledgements This work has been conducted with the use of the UK Biobank Resource under application number 53472.

Author contributions The authors' contributions were as follows: HYB and ELG: designed the research; HYB and FFC: performed the statistical analyses; HYB, FFC and ELG: drafted the manuscript; ELG: supervised the conduct of research and had primary responsibility for final content; and all authors: reviewed the manuscript for important intellectual content, and read and approved the final manuscript. The corresponding author attests that all listed authors meet authorship criteria and that no others meeting the criteria have been omitted.

Funding Open Access funding provided thanks to the CRUE-CSIC agreement with Springer Nature. UK Biobank was established by the Wellcome Trust medical charity, Medical Research Council, Department of Health, Scottish Government and the Northwest Regional Development Agency. It has also had funding from the Welsh Government, British Heart Foundation, Cancer Research UK, and Diabetes UK. This work was supported by FIS grants 19/319 and 20/1040, (Instituto de Salud Carlos III, State Secretary of R+D + I and FEDER/FSE). The funding agencies had no role in the study design, data analysis, interpretation of results, writing of the report, and in the decision to submit the article for publication.

Data availability Data and materials are available if required.

Code availability Code is available if required.

\section{Declarations}

Conflict of interest On behalf of all authors, the corresponding author states that there is no conflict of interest.

Ethical approval This study was performed under generic ethical approval obtained by the UK Biobank from the National Health Service National Research Ethics Service (ref 11/NW/0382, 17 June 2011).

Consent to participate All participants provided their written informed consent for participating in the study.

Consent for publication All authors have approved this manuscript for publication.

Open Access This article is licensed under a Creative Commons Attribution 4.0 International License, which permits use, sharing, adaptation, distribution and reproduction in any medium or format, as long as you give appropriate credit to the original author(s) and the source, provide a link to the Creative Commons licence, and indicate if changes were made. The images or other third party material in this article are included in the article's Creative Commons licence, unless indicated otherwise in a credit line to the material. If material is not included in the article's Creative Commons licence and your intended use is not permitted by statutory regulation or exceeds the permitted use, you will need to obtain permission directly from the copyright holder. To view a copy of this licence, visit http://creativecommons.org/licenses/by/4.0/.

\section{References}

1. Wilson BS, Tucci DL, Merson MH, O’Donoghue GM (2017) Global hearing health care: new findings and perspectives. Lancet 390(10111):2503-2515. https://doi.org/10.1016/S0140-6736(17) 31073-5

2. Mick P, Kawachi I, Lin FR (2014) The association between hearing loss and social isolation in older adults. Otolaryngol Neck Surg 150(3):378-384. https://doi.org/10.1177/0194599813518021

3. Lawrence BJ, Jayakody DMP, Bennett RJ, Eikelboom RH et al (2020) Hearing loss and depression in older adults: a systematic review and meta-analysis. Gerontologist 60(3):e137-e154. https:// doi.org/10.1093/geront/gnz009

4. Lin FR, Yaffe K, Xia J, Xue Q-L et al (2013) Hearing loss and cognitive decline in older adults. JAMA Intern Med 173(4):293299. https://doi.org/10.1001/jamainternmed.2013.1868

5. Tseng Y-C, Liu SH-Y, Lou M-F, Huang G-S (2018) Quality of life in older adults with sensory impairments: a systematic review. Qual Life Res 27(8):1957-1971. https://doi.org/10.1007/ s11136-018-1799-2

6. Lee W, Chang Y, Shin H, Ryu S (2020) Hearing loss and risk of overall, injury-related, and cardiovascular mortality: The Kangbuk Samsung Health Study. J Clin Med 9(5):E1415. https://doi.org/10. 3390/jcm9051415

7. Lin HW, Mahboubi H, Bhattacharyya N (2019) Hearing difficulty and risk of mortality. Ann Otol Rhinol Laryngol 128(7):614-618. https://doi.org/10.1177/0003489419834948

8. Genther DJ, Betz J, Pratt S, Kritchevsky SB et al (2015) Association of hearing impairment and mortality in older adults. J Gerontol A Biol Sci Med Sc 70(1):85-90. https://doi.org/10.1093/ gerona/glu094

9. Nolan LS (2020) Age- related hearing loss: why we need to think about sex as a biological variable. J Neurosci Res 00:1-16. https:// doi.org/10.1002/jnr.24647

10. Puga AM, Pajares MA, Varela-Moreiras G, Partearroyo T (2018) Interplay between Nutrition and Hearing Loss: State of Art. Nutrients 11(1):35. https://doi.org/10.3390/nu11010035

11. Jung SY, Kim SH, Yeo SG (2019) Association of nutritional factors with hearing loss. Nutrients 11(2):307. https://doi.org/10. 3390/nu11020307

12. Houston DK, Johnson MA, Nozza RJ, Gunter EW et al (1999) Age-related hearing loss, vitamin B-12, and folate in elderly women. Am J Clin Nutr 69(3):564-571. https://doi.org/10.1093/ ajen/69.3.564

13. Choi Y-H, Miller JM, Tucker KL, Hu H et al (2013) Antioxidant vitamins and magnesium and the risk of hearing loss in the US general population. Am J Clin Nutr 99(1):148-155. https://doi. org/10.3945/ajen.113.068437

14. Curhan SG, Stankovic KM, Eavey RD, Wang M et al (2015) Carotenoids, vitamin A, vitamin C, vitamin E, and folate and risk of self-reported hearing loss in women. Am J Clin Nutr 102(5):1167-1175. https://doi.org/10.3945/ajcn.115.109314

15. Dawes P, Cruickshanks KJ, Marsden A, Moore DR et al (2020) Relationship between diet, tinnitus, and hearing difficulties. Ear Hear 41(2):289-299. https://doi.org/10.1097/AUD.0000000000 000765

16. Gopinath B, Flood VM, Rochtchina E, McMahon CM et al (2010) Consumption of omega-3 fatty acids and fish and risk of agerelated hearing loss. Am J Clin Nutr 92(2):416-421. https://doi. org/10.3945/ajen.114.091819

17. Dullemeijer C, Verhoef P, Brouwer IA, Kok FJ et al (2010) Plasma very long-chain $n-3$ polyunsaturated fatty acids and age-related hearing loss in older adults. J Nutr Health Aging 14(5):347-351. https://doi.org/10.1007/s12603-010-0078-x 
18. Curhan SG, Eavey RD, Wang M, Rimm EB et al (2014) Fish and fatty acid consumption and the risk of hearing loss in women. Am J Clin Nutr 100(5):1371-1377. https://doi.org/10.3945/ajcn.114. 091819

19. Kim SY, Sim S, Kim HJ, Choi HG (2015) Low-fat and low-protein diets are associated with hearing discomfort among the elderly of Korea. Br J Nutr 114(10):1711-1717. https://doi.org/10.1017/ S0007114515003463

20. Wang DD, Hu FB (2017) Dietary fat and risk of cardiovascular disease: recent controversies and advances. Annu Rev Nut 37:423-446. https://doi.org/10.1146/annurev-nutr-071816-064614

21. Sandoval-Insausti H, Pérez-Tasigchana RF, López-García E, GarcíaEsquinas E et al (2016) Macronutrients intake and incident frailty in older adults: a prospective cohort study. J Gerontol A Biol Sci Med Sci 71(10):1329-1334. https://doi.org/10.1093/gerona/glw033

22. Arias-Fernández L, Struijk EA, Rodríguez-Artalejo F, Lopez-Garcia E et al (2020) Habitual dietary fat intake and risk of muscle weakness and lower-extremity functional impairment in older adults: a prospective cohort study. Clin Nutr. https://doi.org/10.1016/j.clnu. 2020.03.018

23. Collins R (2012) What makes UK Biobank special? Lancet 379(9822):1173-1174. https://doi.org/10.1016/S0140-6736(12) 60404-8

24. Galante J, Adamska L, Young A, Young H et al (2016) The acceptability of repeat Internet-based hybrid diet assessment of previous 24-h dietary intake: administration of the Oxford WebQ in UK Biobank. Br J Nutr 115(4):681-686. https://doi.org/10.1017/S0007 114515004821

25. Bradbury KE, Young HJ, Guo W, Key TJ (2018) Dietary assessment in UK Biobank: an evaluation of the performance of the touchscreen dietary questionnaire. J Nutr Sci 7:e6. https://doi.org/10.1017/jns. 2017.66

26. Food Standards Agency (2002) McCance and Widdowson's the composition of foods, 6th edn. Royal Society of Chemistry, Cambridge

27. Willett WC (2012) Nutritional epidemiology, 3rd edn. Oxford University Press, Oxford

28. Ho FK, Gray SR, Welsh P, Petermann-Rocha F et al (2020) Associations of fat and carbohydrate intake with cardiovascular disease and mortality: prospective cohort study of UK Biobank participants. BMJ 368:m688. https://doi.org/10.1136/bmj.m688

29. Piernas C, Perez-Cornago A, Gao M, Young H, Pollard Z, Mulligan A, Lentjes M, Carter J, Bradbury K, Key TJ, Jebb SA (2021) Describing a new food group classification system for UK biobank: analysis of food groups and sources of macro- and micronutrients in 208,200 participants. Eur J Nutr. https://doi.org/10.1007/ s00394-021-02535-x

30. Curhan SG, Halpin C, Wang M, Eavey RD et al (2020) Prospective study of dietary patterns and hearing threshold decline. Am J Epidemiol 189(3):204-214. https://doi.org/10.1093/aje/kwz223

31. Dawes P, Fortnum H, Moore DR, Emsley R et al (2014) Hearing in middle age: a population snapshot of 40-to 69-year olds in the United Kingdom. Ear Hear 35(3):44-51. https://doi.org/10.1097/ AUD.0000000000000010

32. Smits C, Kapteyn TS, Houtgast T (2004) Development and validation of an automatic speech-in-noise screening test by telephone. Int J Audiol 43:15-28. https://doi.org/10.1080/14992020400050004

33. Keidser G, Seeto M, Rudner M, Hygge S et al (2015) On the relationship between functional hearing and depression. Int J Audiol 54:653-664. https://doi.org/10.3109/14992027.2015.1046503

34. Sudlow C, Gallacher J, Allen N, Beral V et al (2015) UK Biobank: an open access resource for identifying the causes of a wide range of complex diseases of middle and old age. PLoS Med 12(3):e1001779. https://doi.org/10.1371/journal.pmed.1001779

35. Wade KH, Carslake D, Sattar N, Davey Smith G et al (2018) BMI and mortality in UK Biobank: revised estimates using Mendelian randomization. Obesity (Silver Spring) 26(11):1796-1806. https:// doi.org/10.1002/oby.22313

36. Craig CL, Marshall AL, Sjöström M, Bauman AE et al (2003) International physical activity questionnaire: 12-country reliability and validity. Med Sci Sports Exerc 35(8):1381-1395. https://doi.org/10. 1249/01.MSS.0000078924.61453.FB

37. Jakobsen LH, Sorensen JM, Rask IK, Jensen BS et al (2011) Validation of reaction time as a measure of cognitive function and quality of life in healthy subjects and patients. Nutrition 27(5):561-570. https://doi.org/10.1016/j.nut.2010.08.003

38. Perperoglou A, Sauerbrei W, Abrahamowicz M, Schmid M (2019) A review of spline function procedures in R. BMC Med Res Methodol 19(1):46. https://doi.org/10.1186/s12874-019-0666-3

39. Gates GA, Mills JH (2005) Presbycusis. Lancet 366(9491):1111-1120

40. Oosterloo BC, Homans NC, Goedegebure A (2020) Tinnitus affects speech in noise comprehension in individuals with hearing loss. Otol Neurotol. https://doi.org/10.1097/MAO.0000000000002733

41. Hu H, Tomita K, Kuwahara K, Yamamoto M et al (2020) Obesity and risk of hearing loss: a prospective cohort study. Clin Nutr 39(3):870-875. https://doi.org/10.1016/j.clnu.2019.03.020

42. Cunningham LL, Tucci DL (2017) Hearing loss in adults. N Engl J Med 377(25):2465-2473. https://doi.org/10.1056/NEJMra1616601

43. Nakashima T, Naganawa S, Sone M, Tominaga M et al (2003) Disorders of cochlear blood flow. Brain Res Brain Res Rev 43(1):17-28. https://doi.org/10.1016/s0165-0173(03)00189-9

44. Orlando MS, Dziorny AC, Love T, Harrington D et al (2020) Association of audiometric measures with plasma long chain polyunsaturated fatty acids in a high-fish eating population: The Seychelles Child Development Study. Neurotoxicology 77:137-144. https://doi. org/10.1016/j.neuro.2020.01.005

45. Shin SA, Lyu AR, Jeong SH, Kim TH et al (2019) Acoustic trauma modulates cochlear blood flow and vasoactive factors in a rodent model of noise-induced hearing loss. Int J Mol Sci 20(21):5316. https://doi.org/10.3390/ijms20215316

46. Wang J, Liu M, Sung V, Lycett K et al (2020) Associations of retinal vessel caliber with hearing status in childhood and midlife: a crossgenerational population-based study. JAMA Otolaryngol Neck Surg 146(4):323-330. https://doi.org/10.1001/jamaoto.2019.4484

47. Lauretani F, Semba RD, Bandinelli S, Miller ER III et al (2008) Plasma polyunsaturated fatty acids and the decline of renal function. Clin Chem 54(3):475-481. https://doi.org/10.1373/clinchem.2007. 095521

48. Delhez A, Lefebvre P, Péqueux C, Malgrange B et al (2020) Auditory function and dysfunction: estrogen makes a difference. Cell Mol Life Sci 77(4):619-635. https://doi.org/10.1007/ s00018-019-03295-y

49. Krizman J, Skoe E, Kraus N (2012) Sex differences in auditory subcortical function. Clin Neurophysiol 123(3):590-597. https://doi. org/10.1016/j.clinph.2011.07.037

50. Wang DD, Hu FB (2018) Precision nutrition for prevention and management of type 2 diabetes. Lancet Diabetes Endocrinol 6(5):416426. https://doi.org/10.1016/S2213-8587(18)30037-8

51. Ahadi M, Pourbakht A, Jafari AH, Shirjian Z et al (2014) Gender disparity in subcortical encoding of binaurally presented speech stimuli: an auditory evoked potentials study. Auris Nasus Larynx 41(3):239-243. https://doi.org/10.1016/j.anl.2013.10.010

52. Sanders TAB (2014) Protective effects of dietary PUFA against chronic disease: evidence from epidemiological studies and intervention trials. Proc Nutr Soc 73(1):73-79. https://doi.org/10.1017/ S0029665113003789

53. Zong G, Li Y, Sampson L, Dougherty LW et al (2018) Monounsaturated fats from plant and animal sources in relation to risk of coronary heart disease among US men and women. Am J Clin Nutr 107(3):445-453. https://doi.org/10.1093/ajcn/nqx004 
54. Lopez-Garcia E, Schulze MB, Manson JE, Meigs JB, Albert CM, Rifai N, Willett WC, Hu FB (2004) Consumption of (n-3) fatty acids is related to plasma biomarkers of inflammation and endothelial activation in women. J Nutr 134(7):1806-1811. https://doi.org/10. 1093/jn/134.7.1806
55. Seah JY, Gay GM, Su J, Tai ES, Yuan JM, Koh WP, Ong CN, van Dam RM (2017) Consumption of red meat, but not cooking oils high in polyunsaturated fat, is associated with higher arachidonic acid status in Singapore Chinese adults. Nutrients 9(2):101. https:// doi.org/10.3390/nu9020101 